\title{
SnS Quantum Dots as Hole Transporter of Perovskite Solar Cells
}

Yang Li, $, \uparrow, \S,, \|$ Zaiwei Wang, ${ }^{\perp}$ Dan Ren, ${ }^{\S}$ Yuhang Liu, ${ }^{\S}$ Aibin Zheng, ${ }^{\dagger}$ Shaik Mohammed Zakeeruddin,,$^{\S}$ Xiandui Dong,,$^{\ddagger}$ Anders Hagfeldt, ${ }^{\perp}$ Michael Grätzel, ${ }^{\S}$ and Peng Wang*,†

†Department of Chemistry, Zhejiang University, Hangzhou 310028, China

¥Changchun Institute of Applied Chemistry, Chinese Academy of Sciences, Changchun 130022, China

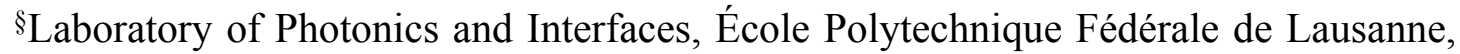
CH-1015 Lausanne, Switzerland

"University of Chinese Academy of Sciences, Beijing 100049, China

${ }^{\perp}$ Laboratory of Photomolecular Science, École Polytechnique Fédérale de Lausanne, CH-1015 Lausanne, Switzerland

*Correspondence to: pw2015@,zju.edu.cn. 

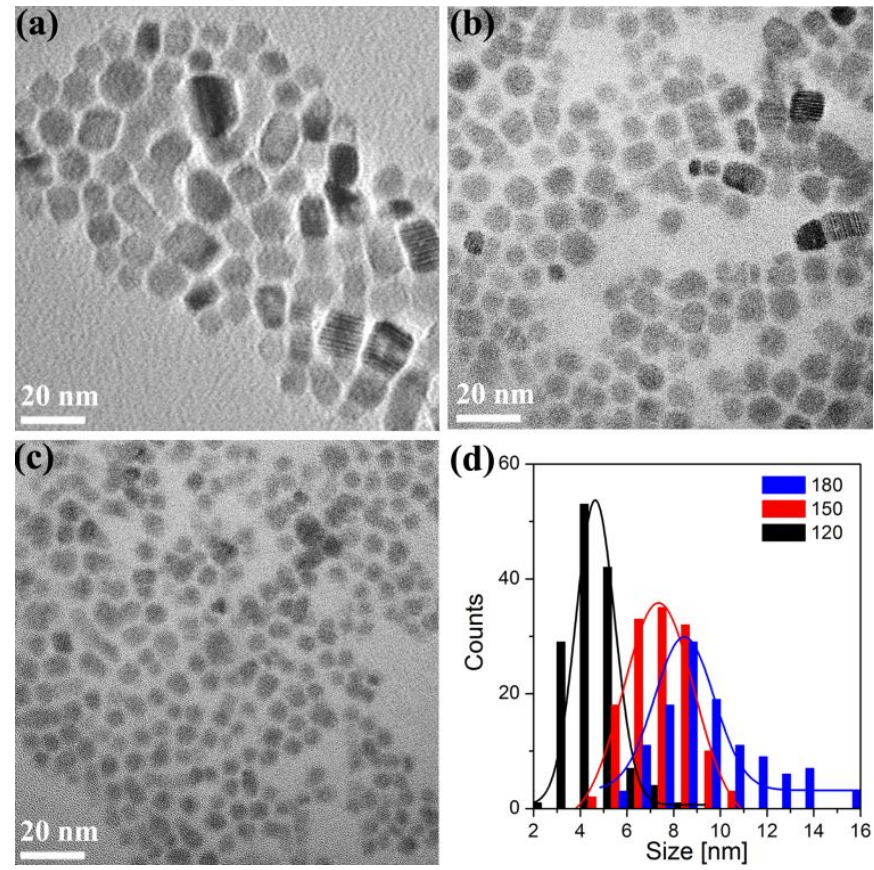

Figure S1. TEM images of the as-synthesized SnS QDs fabricated at different injection temperatures (a) $180{ }^{\circ} \mathrm{C}$, (b) $150^{\circ} \mathrm{C}$ and (c) $120^{\circ} \mathrm{C}$. (d) Size histogram of the different SnS samples. Note that, the SnS QDs synthesized at higher temperature (over $210{ }^{\circ} \mathrm{C}$ ) cannot be dispersed well into chlorobenzene, due to the poor coverage of the bulky ligand, which limits the possibility for further solution processing of this type of dots. 
(a)

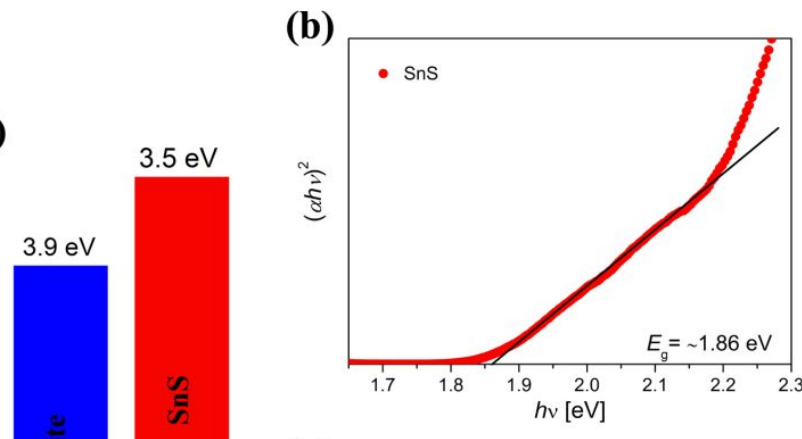

(c)

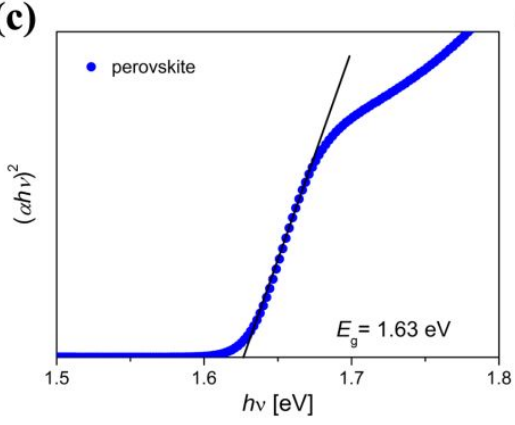

(d)

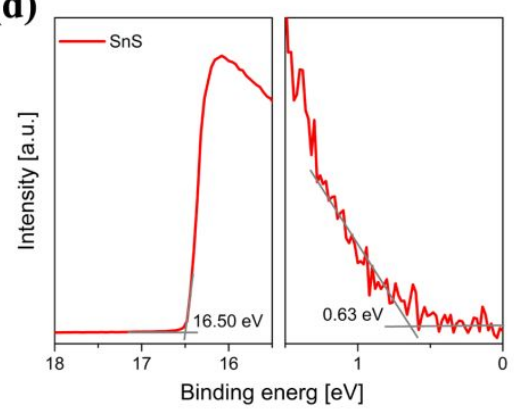

(e)

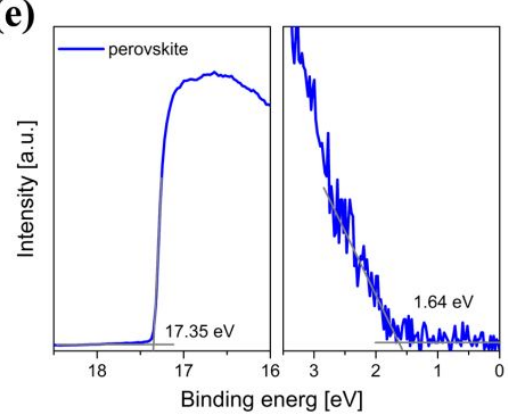

Figure S2. (a) Energy level diagram of a triple cation perovskite and SnS. The conduction band of perovskite was calculated through $E_{C B}=E_{V B}-E_{\mathrm{g}}$. (b) Tauc plot of the SnS QD film. The band gap of 8-nm-sized SnS QDs $(\sim 1.86 \mathrm{eV})$ is larger than the bulk $\operatorname{SnS}(\sim 1.3 \mathrm{eV})$, due to quantum confinement rather than the higher band gap of the cubic phase. (c) Tauc plot of the pristine perovskite film. The Tauc plots were derived from the transmission and reflection spectra of the films with an integrate sphere. UPS spectra of our SnS (d) and perovskite (e) samples in the cutoff energy regions and onset energy regions. The valence bands can be calculated through the equation $E_{V B}=21.21-\left(E_{\text {cutoff }}-E_{\text {onset }}\right)$. 
Table S1 Fitting parameters of TRPL curves for perovskite and perovskite/HTM films

\begin{tabular}{ccccccc}
\hline & $A_{0}$ & $A_{1}$ & $\tau_{1}[\mathrm{~ns}]$ & $A_{2}$ & $\tau_{2}[\mathrm{~ns}]$ & $\tau_{\mathrm{av}}{ }^{\mathrm{a}}[\mathrm{ns}]$ \\
\hline Perovskite & 0.014 & 0.147 & 159 & 0.839 & 1253 & 1075 \\
Perovskite+SnS & 0.003 & 0.991 & 26 & 0.006 & 1406 & 34 \\
Perovskite+spiro-OMeTAD & 0.002 & 0.796 & 37 & 0.202 & 170 & 64 \\
\hline
\end{tabular}

a) $\tau_{\mathrm{av}}$ was obtained with eqn. $\tau_{\mathrm{av}}=A_{1} \tau_{1}+A_{2} \tau_{2}$. 


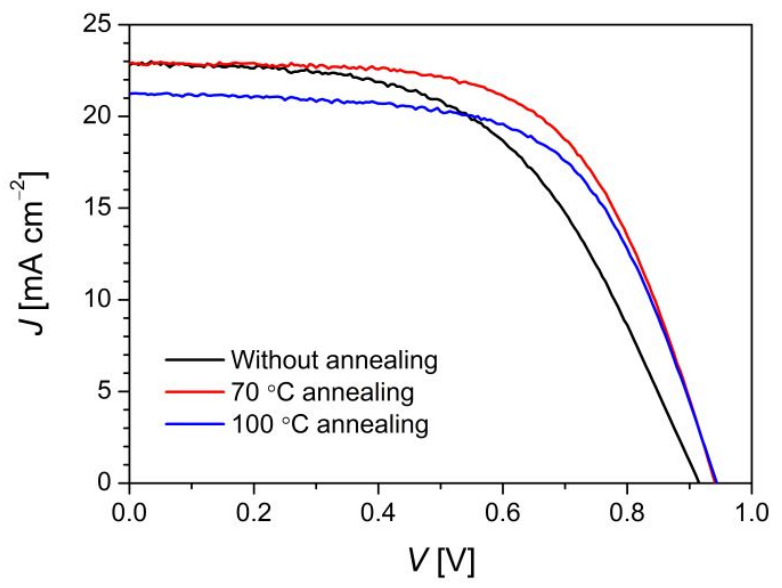

Figure S3. $J-V$ curves of devices at different annealing conditions using the SnS concentration of 20 $\mathrm{mg} \mathrm{ml}^{-1}$ in chlorobenzene. 
Table S2 Photovoltaic parameters obtained from $J-V$ curves in Figure S3

\begin{tabular}{ccccc}
\hline Annealing tem. & $J_{\mathrm{SC}}\left[\mathrm{mA} \mathrm{cm}^{-2}\right]$ & $V_{\mathrm{OC}}[\mathrm{V}]$ & FF & PCE [\%] \\
\hline without & 22.78 & 0.916 & 0.537 & 11.18 \\
$70^{\circ} \mathrm{C}$ & 22.89 & 0.941 & 0.614 & 13.15 \\
$100^{\circ} \mathrm{C}$ & 21.22 & 0.944 & 0.617 & 12.36 \\
\hline
\end{tabular}



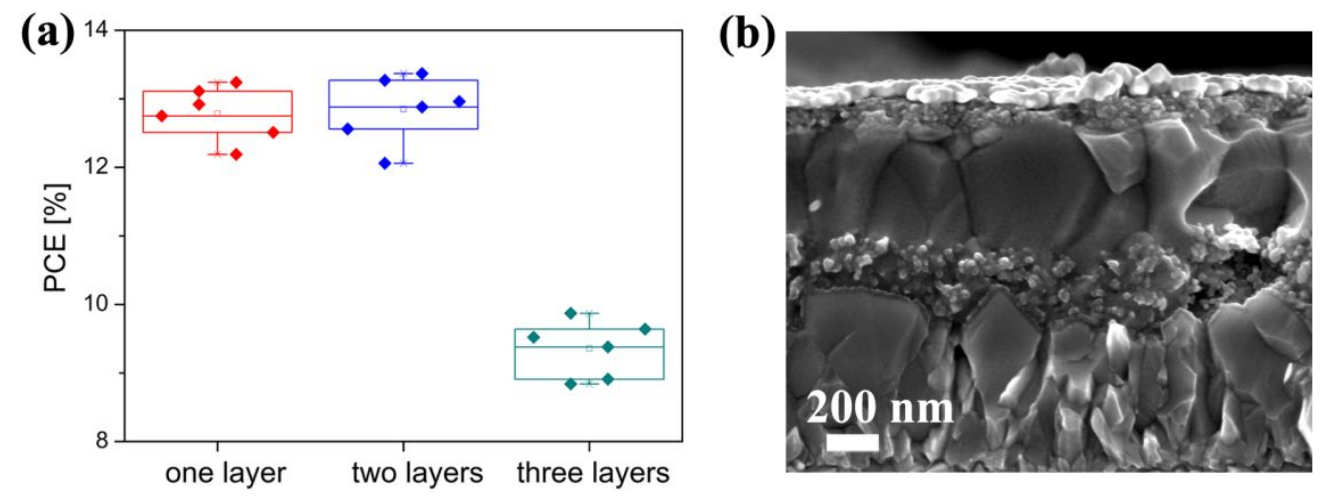

Figure S4. (a) Relationship between the efficiency distribution and the number of SnS layer. The SnS QD films were processed via layer-by-layer deposition, and EDT was adopted to exchange the ligand between each layer. The SnS solution concentration is $20 \mathrm{mg} \mathrm{ml}^{-1}$ for each layer. (b) Cross-sectional SEM image of the device made with two layers of SnS QD film showing the SnS film thickness around $80 \mathrm{~nm}$, which is twice of the thickness of one layer SnS. 


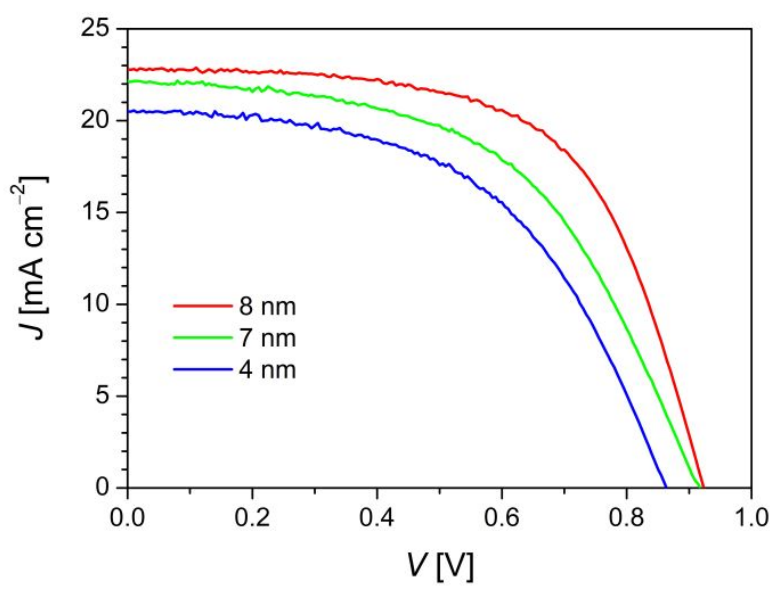

Figure S5. $J-V$ curves of devices based on different size of SnS QDs. 
Table S3 Photovoltaic parameters obtained from $J-V$ curves in Figure S5

\begin{tabular}{ccccc}
\hline Averaged dot size & $J_{\mathrm{SC}}\left[\mathrm{mA} \mathrm{cm}^{-2}\right]$ & $V_{\mathrm{OC}}[\mathrm{V}]$ & $\mathrm{FF}$ & $\mathrm{PCE}[\%]$ \\
\hline $8 \mathrm{~nm}$ & 22.81 & 0.923 & 0.613 & 12.90 \\
$7 \mathrm{~nm}$ & 22.16 & 0.917 & 0.534 & 10.85 \\
$4 \mathrm{~nm}$ & 20.49 & 0.865 & 0.528 & 9.36 \\
\hline
\end{tabular}




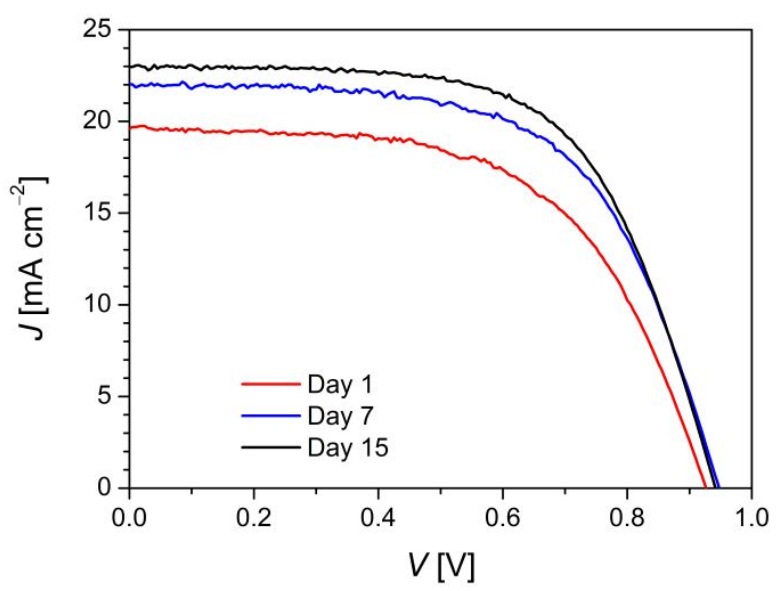

Figure S6. $J-V$ curves of devices based on the $\mathrm{SnS}$ hole transporter measured in dry air over the course of 15 days. 
Table S4 Photovoltaic parameters obtained from the $J-V$ curves in Figure S6

\begin{tabular}{ccccc}
\hline Time & $J_{\mathrm{SC}}\left[\mathrm{mA} \mathrm{cm}^{-2}\right]$ & $V_{\mathrm{OC}}[\mathrm{V}]$ & FF & PCE [\%] \\
\hline Day 1 & 19.60 & 0.927 & 0.583 & 10.59 \\
Day 7 & 22.04 & 0.948 & 0.612 & 12.79 \\
Day 15 & 22.98 & 0.942 & 0.627 & 13.57 \\
\hline
\end{tabular}




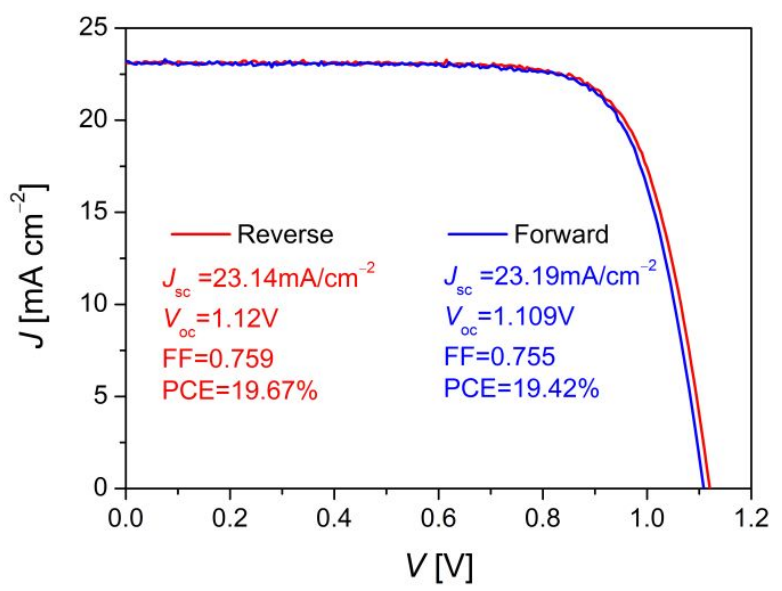

Figure S7. Forward and reverse $J-V$ curves of spiro-OMeTAD based PSCs measured after 5-day aging in the dry-air filled box (relativity humidity $<2 \%$ ). The inset shows the photovoltaic parameters obtained from the corresponding $J-V$ scans. 


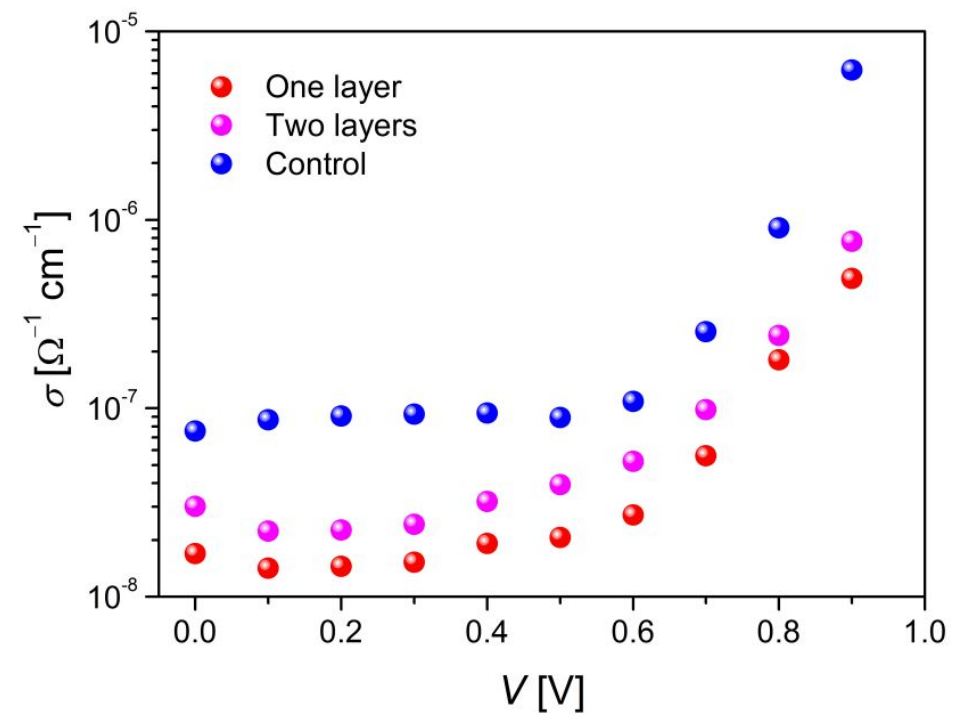

Figure S8. Comparison of the conductivities of hole transporting films derived from impedance analysis. One layer and two layers refer to the SnS films with different deposition circles, and control refers to the spiro-OMeTAD film. 


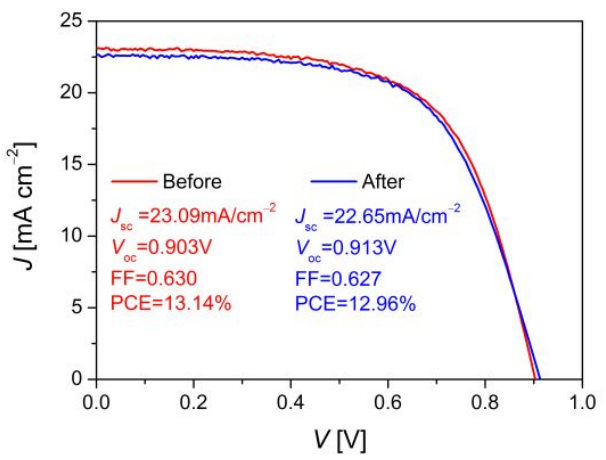

Figure S9. $J-V$ curves of $\mathrm{SnS}$ based perovskite solar cells before and after 1,000 h storage under ambient conditions. The inset shows the photovoltaic parameters obtained from the corresponding $J-V$ curves. 


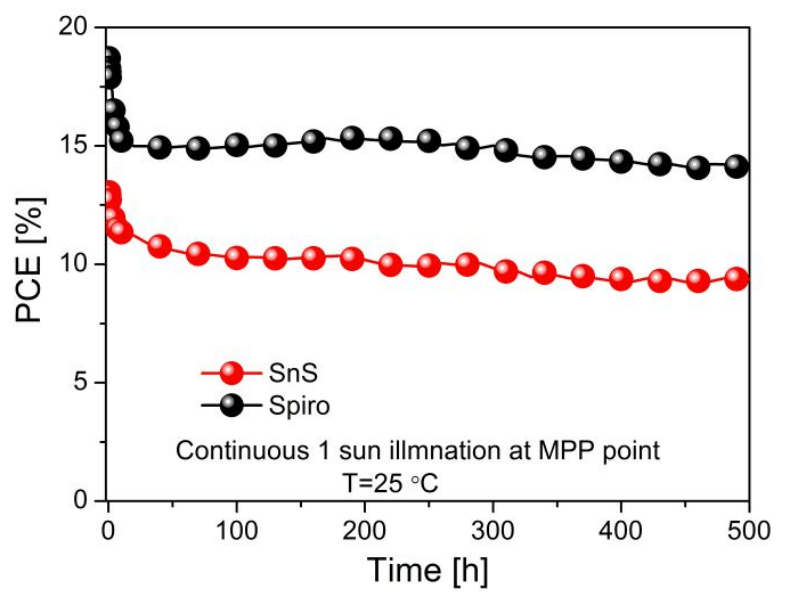

Figure S10. Operation stability of un-encapsulated PSCs with SnS and spiro-OMeTAD as the hole-transporting layers. The device efficiency evolutions were obtained by MPP tracking at $25{ }^{\circ} \mathrm{C}$ under continuous one sun irradiation, in an atmosphere of nitrogen. Here, the efficiency of control devices shows $75 \%$ retention after $500 \mathrm{~h}$ aging, which lies within the range of stability results obtained from our lab as shown in Figure 1 of ref 8. 